\title{
The Proportion of Obstructive Sleep Apnoea in Coronary Artery Disease Patients Using Berlin Questionnaire and STOP-BANG Questionnaire
}

\author{
Juhin Thomas ${ }^{1}$, Saritha J. Shenoy², Geethadevi Madhavikutty ${ }^{3}$ \\ ${ }^{1}$ Government Medical College, Kottayam, Kerala, India. ${ }^{2}$ Department of Physiology, Government Medical College, \\ Kottayam, Kerala, India. ${ }^{3}$ Department of Community Medicine, Government Medical College, Kottayam, Kerala, India.
}

\section{ABSTRACT}

\section{BACKGROUND}

Obstructive Sleep Apnoea (OSA) is a highly prevalent disease but remains underrecognized in India. Polysomnography is the gold standard test in the diagnosis of OSA but it is difficult to perform and not frequently done. Simple questionnairebased diagnostic tools can be included in the screening procedure of patients with coronary artery disease (CAD) to detect the need for further OSA evaluation. The present study was undertaken to find out the proportion of OSA in CAD patients using Berlin's and STOP-BANG questionnaires.

\section{METHODS}

A cross-sectional study was conducted to find out the proportion of OSA in CAD patients among 70 known cases of CAD referred from the Cardiology department of Government Medical College, Kottayam and 70 normal healthy adults as controls using Berlin and STOP-BANG questionnaires and the results were analyzed by SPSS software.

\section{RESULTS}

Among individuals with CAD, $20 \%, 51.4 \%, 28.6 \%$ were found to be at high, intermediate and low risk for OSA whereas $2.9 \%, 31.4 \%, 65.7 \%$ were only found to have a high, intermediate and low risk for OSA in the control population as per STOP-BANG questionnaire. Around $37.1 \%$ and $62.9 \%$ were found to be at high and low risk for OSA among the CAD patients, whereas in the control group $11.4 \%$ and $88.6 \%$ were at high and low risk for OSA respectively. Hence the proportion of OSA (high risk as per the questionnaires) in CAD patients was $20 \%$ and $37.1 \%$ as per STOP-BANG and Berlin questionnaires respectively. The mean score obtained in CAD patients was 3.31 whereas that in normal individuals was 2.04 , using the STOPBANG questionnaire. Similarly, it was 2.97 in CAD patients and 1.11 in normal individuals as per the Berlin questionnaire (P-value $=0.001$ )

\section{CONCLUSIONS}

Increased risk of OSA in CAD patients compared to the normal individuals mean score for OSA was significantly high in CAD patients than in the control group.

\section{KEY WORDS}

OSA, CAD, Polysomnography, STOP BANG Questionnaire, Berlin Questionnaire.
Corresponding Author: Dr. Saritha J. Shenoy, Assistant Professor, Department of Physiology, Government Medical College, Kottayam-686008, Kerala, India. E-mail: ajaikamathsaritha@gmail.com

DOI: 10.14260/jemds/2022/15

How to Cite This Article:

Thomas J, Shenoy SJ, Madhavikutty G. The proportion of obstructive sleep apnoea in coronary artery disease patients using Berlin questionnaire and STOP - BANG questionnaire. J Evolution Med Dent Sci 2022;11(01):78-82, DOI: 10.14260/jemds/2022/15

Submission 26-11-2021,

Peer Review 26-12-2021,

Acceptance 04-01-2022,

Published 25-01-2022.

Copyright (C) 2022 Juhin Thomas et al. This is an open access article distributed under Creative Commons Attribution License [Attribution 4.0 International (CC BY 4.0)] 


\section{BACKGROUND}

Obstructive sleep apnoea (OSA) is a clinical condition characterized by snoring, daytime sleepiness, fatigue, repeated termination of airflow (apnoea) and hypoxemia. OSA is considered as an independent risk factor for various cardiovascular diseases like hypertension, coronary artery disease, stroke and pulmonary hypertension. Moreover, activation of the sympathetic nervous system during respiratory events potentiates vasoconstriction and often triggers an increase in blood pressure and heart rate. ${ }^{1}$ It is one of the most prevalent breathing disturbances in sleep, affecting $2 \%$ to $27 \%$ of the general population. An increased risk for developing postoperative complications, ICU admissions, and increased duration of hospital stay following surgeries in patients with OSA has also been documented. Untreated OSA can lead to increased mortality and morbidity. The underlying mechanisms explaining associations between obstructive sleep apnoea and cardiovascular disease are not entirely delineated. Several intermediary mechanisms might be involved including sustained sympathetic activation, intrathoracic pressure changes, and oxidative stress. ${ }^{2}$

Early diagnosis of OSA is hence, very essential. The gold standard for diagnosis is polysomnography (PSG), which involves overnight monitoring of several physiological variables including electroencephalography, eye movements, muscle tone, respiratory effort, nasal airflow and oxygen saturation. ${ }^{3}$ According to the recently updated International Classification of Sleep Disorders published by the American Academy of Sleep Medicine, OSA is diagnosed when apnoeahypopnea index (AHI) i.e., a total number of obstructive apnoea and hypopnoea per hour of sleep $>5$ and the severity is graded based on clinical criteria as mild ( $\mathrm{AHI}>5$ but $<15$ ), moderate $\left(\mathrm{AHI}>15\right.$ but $<30$ ) and severe $(\mathrm{AHI}>30){ }^{4}$

Coronary Artery Disease (CAD) is also a major health problem and its clinical spectrum ranges from angina pectoris to acute coronary syndromes. It is also a major cause of morbidity and mortality in many countries. There is a high prevalence of sleep apnoea in patients with heart failure (HF), both with reduced and preserved ejection fraction (EF). Earlier studies estimated the prevalence of OSA in heart failure with reduced EF as 12-15\%, while the prevalence of central sleep apnoea (CSA) as between 37-38 \%.5 The association of OSA and CAD is very complex and described as the development of oxidative stress in CAD patients that induce atherosclerosis and poor outcome in patients. More recent studies have found an even higher prevalence of both CSA and OSA in heart failure patients. Out of 126 patients with NYHA classes II-IV HF, 71\% were diagnosed with sleep apnoea of which $65 \%$ had CSA and $35 \%$ OSA. ${ }^{6}$

For the past few years, the diagnosis of OSA using PSG is inaccessible and expensive. An established sleep lab with a trained technician is essential. It has become very difficult for many hospitals to find trained manpower to interpret PSG. In this context, the accuracy of OSA questionnaires has been an area of growing research interest and a number of studies have been published on the subject. Among these the popularly used are the Berlin questionnaire (BQ), STOPBANG questionnaire (SBQ), STOP questionnaire (SQ), and Epworth Sleepiness Scale (ESS). Most popular of them are STOP-BANG and Berlin questionnaires, which are validated and more economical. Such diagnostic tools can be used for screening of OSA in coronary artery disease patients. ${ }^{7}$

\section{Objectives}

- To determine the proportion of obstructive sleep apnea in coronary artery disease patients using Berlin and STOP-BANG questionnaires.

- To compare the mean score of Berlin and STOP-BANG questionnaires in obstructive sleep apnoea in coronary artery disease patients.

\section{METHODS}

A descriptive study was conducted in Cardiology OPD at Government Medical College, Kottayam on 70 diagnosed cases of coronary artery disease referred from cardiology OPD and 70 normal healthy adults as controls. Each subject was provided with STOP-BANG and Berlin questionnaires in which demographic data, health status and consent to participate in the study were recorded. The objectives of the study were explained to the subjects and were familiarized.

\section{Study Duration}

2 months from the date of IRB clearance (17 July $2019-16$ Sept. 2019

\section{Sample Size}

The study was conducted on 70 diagnosed cases of coronary artery disease and 70 normal healthy adults as controls as the minimum required sample size was 69.5

It was calculated by, Sample Size $=\frac{(\mathrm{z} \alpha+\mathrm{z} \beta)^{2} \times 2 \times \mathrm{PxQ}}{\left(P_{1}-P_{2}\right)^{2}}$

$\mathrm{Z} \alpha=1.96$

$\mathrm{Z} \beta=0.84$

$P=\frac{P_{1}+P_{2}}{2} ; \mathrm{Q}=100-\mathrm{P}$

( $\mathrm{P}_{1}$-prevalence of OSA in CAD in India, $\mathrm{P}_{2}$-Prevalence of OSA in the general population)

$P_{1}=56.4 \%$, according to a study conducted in Saudi Arabia ${ }^{8}$

$P_{2}=32.8 \%$, according to a study conducted in Sao Paulo ${ }^{9}$

$\mathrm{P}=44.6, \mathrm{Q}=55.4$

Sample Size $=\frac{(1.96+0.84)^{2} \times 2 \times 44.6 \times 55.4}{(23.6 \times 23.6)^{2}}=69.56=70$.

\section{Sampling Technique}

Convenient Sampling.

\section{Inclusion Criteria}

Known cases of CAD patients in the Cardiology department, Government Medical College, Kottayam \& normal healthy adults in the age group of $20-60 \mathrm{yrs}$. 


\section{Exclusion Criteria}

1. Patients less than 20 yrs \& more than 60 yrs.

2. Patients with any other cardiorespiratory or medical illness.

\section{Questionnaires}

STOP-BANG questionnaire contains mainly 4 subjective items (snoring, tiredness, observed apnoea, high BP) accounting to STOP and 4 demographic items (BMI, age, neck circumference, gender) accounting for BANG. Each question carries a score of one. A score of 5-8 is categorized as high risk for 0SA. ${ }^{10}$ Berlin's questionnaire consisted of 10 questions in three categories. In category one, high risk was defined as persistent symptoms in two or more questions related to snoring. In category two, high risk was defined as persistent daytime sleepiness, drowsy driving, or both. In category three, high risk was defined as a history of hypertension or a BMI higher than $30 \mathrm{~kg} / \mathrm{m}^{2}$. High-risk subjects for OSA were those who were defined as high risk in at least two out of three categories. ${ }^{11}$

\section{Statistical Analysis}

Data were analysed using SPSS software. Categorical variables were analysed using the chi-square test and mean score using the unpaired t-test.

\section{RESULTS}

\section{Physical Parameters of the Subjects}

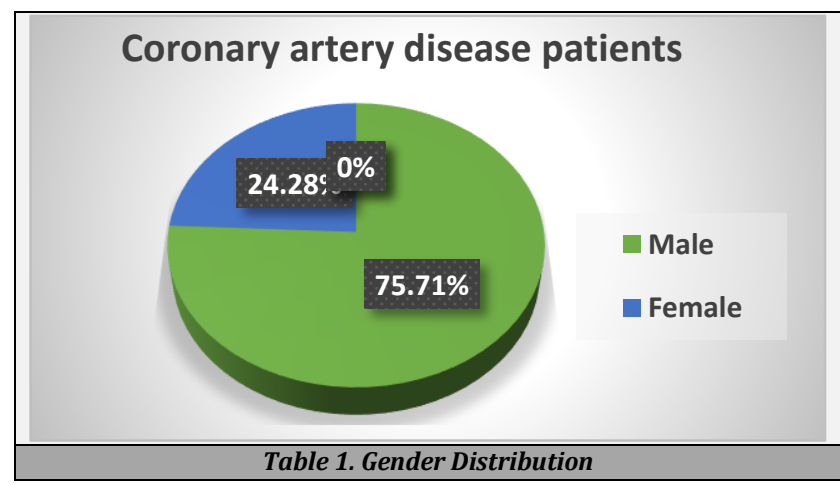

Among CAD patients, $75.71 \%$ were males and the rest were females, whereas males and females were considered in almost equal proportion from the general population.

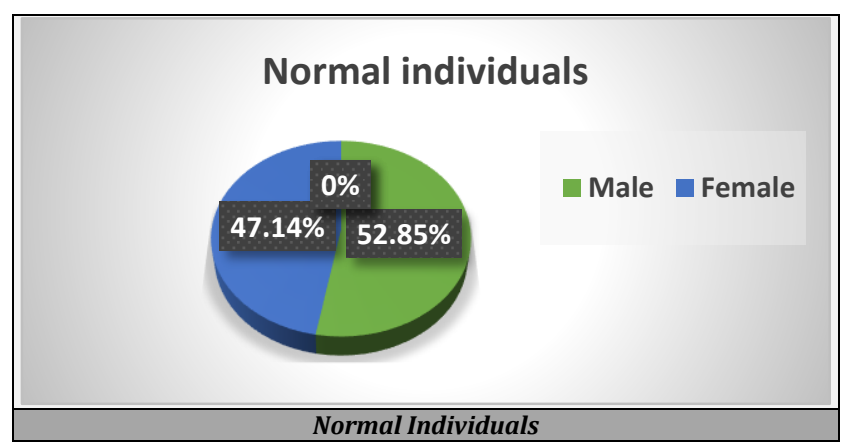

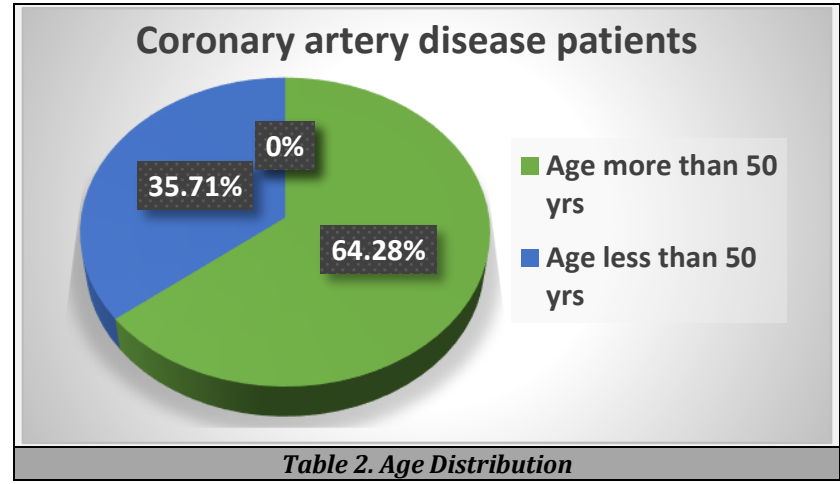

Among CAD patients, $64.28 \%$ were above 50 yrs., of age and $55.71 \%$ of individuals aged more than 50 yrs. were considered from the general population.
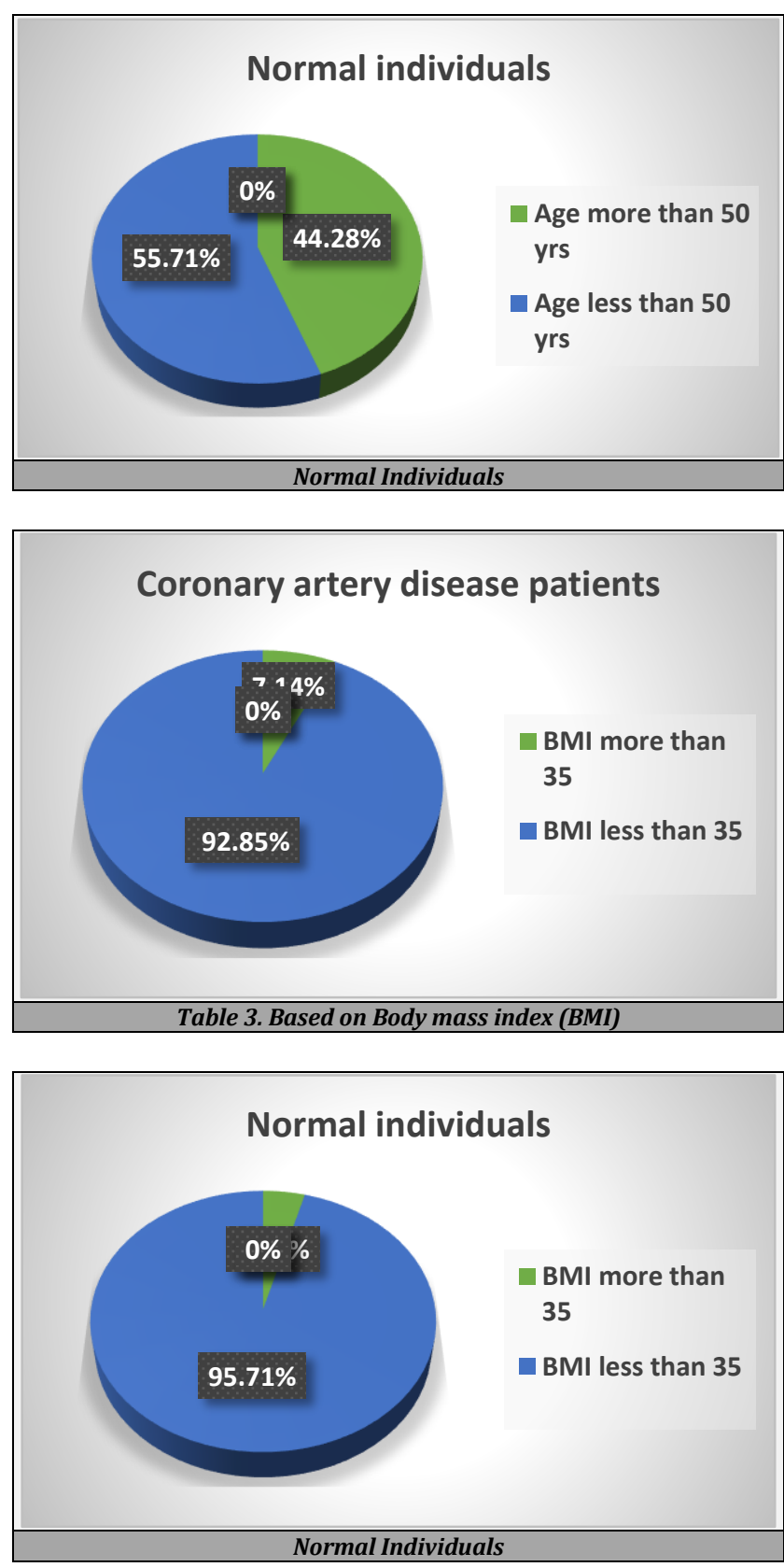

Only $7.14 \%$ of the CAD patients and $4.28 \%$ in the general population considered were with a BMI of more than 35 . 


\begin{tabular}{|c|c|c|c|c|c|}
\hline & & & \multicolumn{2}{|c|}{ Category } & \multirow[b]{2}{*}{ Total } \\
\hline & & & 1 & 2 & \\
\hline \multirow{3}{*}{$\begin{array}{l}\text { Risk as per } \\
\text { STOP BANG }\end{array}$} & 1 & $\begin{array}{c}\text { Count } \\
\% \text { within CATEGORY }\end{array}$ & $\begin{array}{c}14 \\
20.0 \%\end{array}$ & $\begin{array}{c}2 \\
2.9 \%\end{array}$ & $\begin{array}{c}16 \\
11.4 \%\end{array}$ \\
\hline & 2 & $\begin{array}{c}\text { Count } \\
\% \text { within CATEGORY }\end{array}$ & $\begin{array}{c}36 \\
51.4 \%\end{array}$ & $\begin{array}{c}22 \\
31.4 \%\end{array}$ & $\begin{array}{c}58 \\
41.4 \%\end{array}$ \\
\hline & 3 & $\begin{array}{c}\text { Count } \\
\% \text { within CATEGORY }\end{array}$ & $\begin{array}{c}20 \\
28.6 \%\end{array}$ & $\begin{array}{c}46 \\
65.7 \%\end{array}$ & $\begin{array}{c}66 \\
47.1 \%\end{array}$ \\
\hline \multirow[t]{2}{*}{ Total } & & $\begin{array}{c}\text { Count } \\
\% \text { within CATEGORY }\end{array}$ & $\begin{array}{c}70 \\
100.0 \%\end{array}$ & $\begin{array}{c}70 \\
100.0 \%\end{array}$ & $\begin{array}{c}140 \\
100.0 \%\end{array}$ \\
\hline & \multicolumn{2}{|c|}{ Chi-square value } & & 22.622 & \\
\hline
\end{tabular}

The risk obtained as per the STOP BANG questionnaire in CAD patients was found to be statistically significant with a $\mathrm{p}$ value of 0.001 and a chi-square value of 22.622. Hence it points to an increased risk of obstructive sleep apnoea in coronary artery disease patients when compared to the control population that is normal individuals.

\begin{tabular}{|c|c|c|c|c|c|}
\hline & & & & ory & \\
\hline & & & 1 & 2 & Total \\
\hline Risk as per & 1 & $\begin{array}{c}\text { Count } \\
\% \text { within CATEGORY }\end{array}$ & $\begin{array}{c}26 \\
37.1 \%\end{array}$ & $\begin{array}{c}8 \\
11.4 \%\end{array}$ & $\begin{array}{c}34 \\
24.3 \%\end{array}$ \\
\hline Berlin & 2 & $\begin{array}{c}\text { Count } \\
\% \text { within CATEGORY }\end{array}$ & $\begin{array}{c}44 \\
62.9 \%\end{array}$ & $\begin{array}{c}62 \\
88.6 \%\end{array}$ & $\begin{array}{c}106 \\
75.7 \%\end{array}$ \\
\hline Total & & $\begin{array}{c}\text { Count } \\
\% \text { within CATEGORY }\end{array}$ & $\begin{array}{c}70 \\
100.0 \%\end{array}$ & $\begin{array}{c}70 \\
100.0 \%\end{array}$ & $\begin{array}{c}140 \\
100.0 \%\end{array}$ \\
\hline & & quare value & & 12.586 & \\
\hline & & o-value & & 0.001 & \\
\hline Table & & of OSA in CAD Pati & as Pe & in Ques & aaire \\
\hline
\end{tabular}

Risk among CAD patients was found to be statistically significant with a P-value of 0.001 and a chi-square value of 12.586, pointing to an increased risk of OSA in CAD patients when compared to the normal individuals as per the Berlin questionnaire.

\begin{tabular}{|c|c|c|c|c|c|c|c|}
\hline & Category & $\mathbf{N}$ & Mean & $\begin{array}{c}\text { Std. } \\
\text { Deviation }\end{array}$ & $\begin{array}{l}\text { Std. } \\
\text { Error } \\
\text { Mean }\end{array}$ & $\begin{array}{c}\mathrm{t}- \\
\text { value }\end{array}$ & $\begin{array}{c}\text { P- } \\
\text { Value }\end{array}$ \\
\hline Total & 1 & 70 & 3.31 & 1.420 & .170 & 5.611 & 0.001 \\
\hline Score & 2 & 70 & 2.04 & 1.256 & .150 & & \\
\hline \multicolumn{8}{|c|}{ Table 6. Mean Score of OSA as Per STOP-BANG Questionnaire } \\
\hline
\end{tabular}

\begin{tabular}{|lccccccc|}
\hline & Category & N & Mean & $\begin{array}{c}\text { Std. } \\
\text { Deviation }\end{array}$ & $\begin{array}{c}\text { Std. } \\
\text { Error } \\
\text { Mean }\end{array}$ & $\begin{array}{c}\text { t- } \\
\text { value }\end{array}$ & $\begin{array}{c}\text { P- } \\
\text { Value }\end{array}$ \\
Total & 1 & 70 & 2.97 & 2.043 & .244 & 5.559 & 0.001 \\
Score & 2 & 70 & 1.11 & 1.908 & .228 \\
\hline \multicolumn{7}{|c|}{ Table 7. Mean Score of OSA as Per Berlin Questionnaire } \\
\hline \\
The mean score obtained was found to be statistically significant with a t-value of \\
5.559 and a p-value of 0.001.
\end{tabular}

\section{DISCUSSION}

Obstructive sleep apnoea (OSA) is a highly prevalent sleep disorder; however, it remains underdiagnosed and undertreated. OSA is associated with various cardiovascular diseases (CVD). An increased risk for developing postoperative complications, ICU admissions, and increased duration of hospital stay following surgeries in patients with OSA has also been documented. Untreated OSA can lead to increased morbidity and mortality. ${ }^{2}$

Early diagnosis of OSA is hence essential. The gold standard for the diagnosis is polysomnography (PSG), which involves overnight monitoring of several physiological variables. Because of the high cost and inaccessibility of PSG, various standard screening tools are now available.
We have undertaken a study to find out the proportion of OSA in CAD patients using two such standard questionnaires, i.e. STOP-BANG and Berlin questionnaires.

The study was conducted on 70 diagnosed cases of CAD and 70 normal healthy adults as controls. The mean age of individuals with coronary artery disease was found to be 54.53 with a standard deviation of 5.5 , whereas the mean age of individuals in the control population was 47.01 with a standard deviation of 10.85 owing to the mean age of the total study population as 50.77 with a standard deviation of 9.363. Among which $64.28 \%$ of individuals with coronary artery disease were aged above 50 yrs, whereas only $35.71 \%$ of them with coronary artery disease were aged less than 50 yrs. This male predominance might be because male gender is also attributed as a non-modifiable risk factor for the development of $\mathrm{CAD}$ along with which more male CAD patients have approached the OP during our study period.

Among the individuals with coronary artery disease around $20 \%, 51.4 \%, 28.6 \%$ were found to be at high, intermediate and low risk for obstructive sleep apnoea, whereas $2.9 \%, 31.4 \%, 65.7 \%$ were only found to have a high, intermediate and low risk for obstructive sleep apnoea in the control population as per STOP-BANG questionnaire. Around $37.1 \%$ and $62.9 \%$ were found to be at high and low risk for obstructive sleep apnoea among the CAD patients, whereas in the control group $11.4 \%$ and $88.6 \%$ were at high and low risk for OSA respectively. Hence, the proportion of OSA (high risk as per the questionnaires) in CAD patients was $20 \%$ as per STOP-BANG and $37.1 \%$ as per Berlin questionnaires, whereas among normal individuals, it was 2.9 $\%$ and $11.4 \%$ as per STOP-BANG and Berlin questionnaires respectively, pointing to an increased risk of OSA in CAD patients as per STOP-BANG and Berlin questionnaires when compared to the normal individuals.

The mean score obtained in our study on calculating the proportion of obstructive sleep apnoea in coronary artery disease patients was 3.31 whereas that in normal individuals was 2.04, using the STOP-BANG questionnaire. Similarly, the mean score obtained was 2.97 in CAD patients and that for normal individuals was 1.11 as per the Berlin questionnaire. Therefore, it has been found that the mean score for OSA was significantly high in CAD patients as compared to the control group based on STOP-BANG and Berlin questionnaires.

Based on the risk of OSA assessed in CAD patients, individuals who were identified to be at a high risk depending on the two questionnaires shall only be directed for PSG for further OSA evaluation and confirmation so that these screening tools may help to reduce the burden for PSG.

\section{CONCLUSIONS}

In our study, to determine the proportion of OSA, among the individuals with coronary artery disease around $20 \%, 51.4$ $\%, 28.6 \%$ were found to be at high, intermediate, and low risk for obstructive sleep apnoea whereas $2.9 \%, 31.4 \%, 65.7$ $\%$ were only found to have high, intermediate and low risk for obstructive sleep apnoea in the control population as per STOP BANG questionnaire. Around $37.1 \%$ and $62.9 \%$ were found to be at high and low risk for obstructive sleep apnoea among the CAD patients, whereas in the control group $11.4 \%$ and $88.6 \%$ were at high and low risk for OSA respectively. 
Hence, the proportion of OSA (high risk as per the questionnaires) in CAD patients was $20 \%$ as per STOP-BANG and $37.1 \%$ as per Berlin questionnaires whereas among normal individuals, it was $2.9 \%$ and $11.4 \%$ as per STOPBANG and Berlin questionnaires respectively, pointing to an increased risk of OSA in CAD patients as per STOP BANG and Berlin questionnaires when compared to the normal individuals.

The mean score obtained on calculating the proportion of obstructive sleep apnoea in coronary artery disease patients was 3.31, whereas in normal individuals it was 2.04, using STOP BANG questionnaire. Similarly, the mean score obtained was 2.97 in CAD patients and that for normal individuals was 1.11 as per Berlin questionnaire. Therefore, it has been found that mean score for OSA was significantly high in CAD patients as compared to the control group based on STOP BANG and Berlin questionnaires.

The risk of OSA in CAD patients was assessed and compared with the normal healthy adults using both STOP BANG and Berlin questionnaires. Using the obtained data, individuals at a high risk for OSA were directed for PSG for further evaluation and confirmation, so that they are adequately treated and prevented from entering into further complications along with reducing the burden for PSG which in turn is a cumbersome procedure.

Data sharing statement provided by the authors is available with the full text of this article at jemds.com.

Financial or other competing interests: None.

Disclosure forms provided by the authors are available with the full text of this article at jemds.com.

ICMR, Department of Cardiology, GMC, Kottayam, Department of Physiology, GMC Kottayam, Department of Community Medicine, GMC Kottayam

\section{REFERENCES}

[1] Netzer NC, Stoohs RA, Netzer CM, et al. Using the Berlin Questionnaire toidentify patients at risk for the sleep apnea syndrome. Ann Intern Med 1999;131(7):485-91.

[2] Kaw R, Pasupuleti V, Walker E, et al. Postoperative complications in patients with obstructive sleep apnea. Chest 2012;141(2):436-41.

[3] Corral-Penafiel J, Jean-Louis P, Barbe F. Ambulatory monitoring in the diagnosis and management of obstructive sleep apnoea syndrome. Eur Respir Rev 2013;22(129):312-24.

[4] Chung F, Yang Y, Liao P. Predictive performance of the STOP-Bang score for identifying obstructive sleep apnea in obese patients. Obes Surg 2013;23(12):2050-7.

[5] De Torres-Alba F, Gemma D, Armada-Romero E, et al. Obstructive sleep apnea and coronary artery disease: from pathophysiology to clinical implication. Pulmonary Medicine 2013;2013:1-9.

[6] Savransky V, Nanayakkara A, Li J, et al. Chronic intermittent hypoxia induces atherosclerosis. Am J Respir Crit Care Med 2007;175(12):1290-7.

[7] Chung F, Chau E, Yang Y, et al. Serum bicarbonate level improves specificity of STOP-Bang screening for obstructive sleep apnea. Chest 2013;143(5):1284-93.

[8] Wali SO, Khali BA, Krayam A. Prevalence and risk factors of obstructive sleep apnea syndrome in a Saudi Arabian population. Ann Thorac Med 2017;12(2):88-94.

[9] Tufik S, Santos-Silva R, Taddei JA, et al. Obstructive Sleep apnea syndrome in the Sao Paulo epidemiologic sleep study. Sleep Med 2010;11(5):441-6.

[10] Chung F, Subramayan R, Liao P, et al. High STOP-Bang score indicates a high probability of obstructive sleep apnoea. Br J Anaesth 2012;108(5):768-75.

[11] Tan A, Yin JDC, Tan LWL, et al. Using the Berlin questionnaire to predict obstructive sleep apnea in the general population. J Clin Sleep Med 2017;13(3):427-32. 